\title{
Analysis and Experimental Study of Median- Nonlinear Flow Characteristics in Hydraulic Proportional Valve
}

\author{
Wenhua Jia \\ School of Mechanical Engineering \\ Nanjing Institute of Technology \\ Nanjing, China \\ E-mail:geovrml@163.com
}

\author{
Chenbo Yin * \\ Institute of Automobile and Construction Machinery \\ Nanjing University of Technology \\ Nanjing, China \\ E-mail: yinchenbo@njtech.edu.cn \\ * Corresponding Author
}

\author{
Dasheng Zhu \\ School of Mechanical Engineering \\ Nanjing Institute of Technology \\ Nanjing, China \\ E-mail: zdssky@163.com \\ Guo Li \\ School of Mechanical Engineering \\ Nanjing Institute of Technology \\ Nanjing, China \\ E-mail: liguoperfect@163.com
}

\begin{abstract}
The nonlinear flow characteristics induced by the geometrical features in various flow areas are often ignored. The strictly dynamical symmetry assumption is not sufficient to design the hydraulic control system by adopting the proportional valve, and the research about the proportional valve model can be used in all moving areas and all covered types of spool is deficient. To overcome this problem, the uniform nonlinear flow mathematical model for the electrohydraulic proportional valve is constructed. The relational expression between the orifice flow of the proportional valve and the geometrical future and physical characteristics of spool is constructed to obtain a series continuous nonlinear flow equations of median positive overlap, negative overlap and zero overlap proportional valve. The experiment validates that, for positive overlap, the flow input into the hydraulic tank is proportional to the displacement of the spool in the dead zone. For negative overlap, even though the spool located at the median, the flow output also exists, and induces the piston movement of hydraulic tank. This situation is consistent with the non-matching overlap response of actual negative proportional valve.
\end{abstract}

Keywords- Proportional valve; median overlap; flow control; nonlinear flow; nonmatching response

\section{INTRODUCTION}

When the spool of the proportional valve is located at the median position, the small change of the overlap value will have great influence to the dy-namic characteristics. For example, because of the dead zone characteristic of flow gain, the positive overlap spool will induce steady state error[1]. The strictly dynamical symmetry assumption is not sufficient to design the hydraulic control system by adopting the proportional valve, and the research about the proportional valve model which can be used in all the moving areas and all covered types of spool is deficient. However, a more comprehensive proportional valve model is necessary to design and analyze the similarity system. A variety of proportional valve mathematic model[2,3,4] have described the complexity transformation relationship of the proportional valve electric, proportional valve machine and proportional valve liquid, but did not describe the nonlinear flow characteristics induced by the geometrical feature of valve in the variety flow zone.

This paper establishes the uniform nonlinear mathematical model. which is related to the relationship between the flow of port and the geometrical characteristics and physical variables of the spool. The aim of this research is to obtain the flow equation when the spool is positive overlap, negative overlap and zero overlap, and the flow is expressed as the continuous and nonlinear function of spool overlap parameters.

Fig.1 shows a big flow proportional valve which is used in the excavator. The proportional valve has two ports named port1 and port 2, each of which has three corresponding flows $Q_{1}, Q_{1 P}, Q_{1 T}$ and $Q_{2}, Q_{2 P}, Q_{2 T}$, respectively. The oil inlet and return are connected with the pump and tank, respectively. The parameters $d$ and $d t$ represent the positive overlap and negative overlap when the spool is located at the median (positive overlap is shown in Fig.1). Fig.2 shows the calculation model of flow field of valve chamber. 


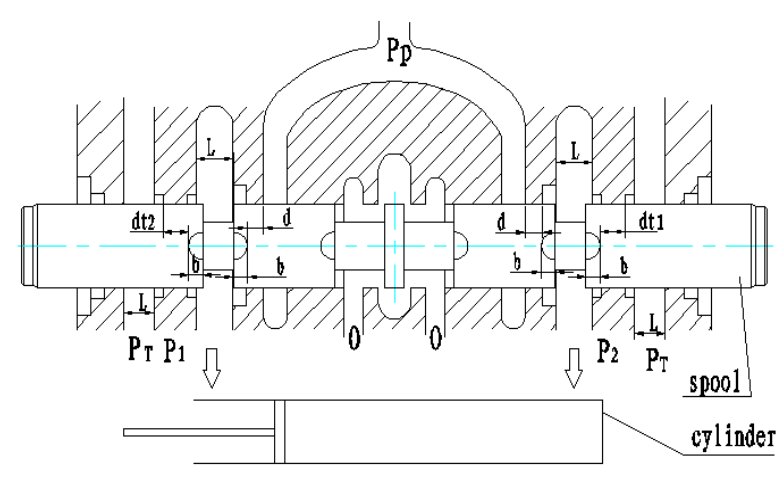

Figure 1. Struture of large flow proportional valve

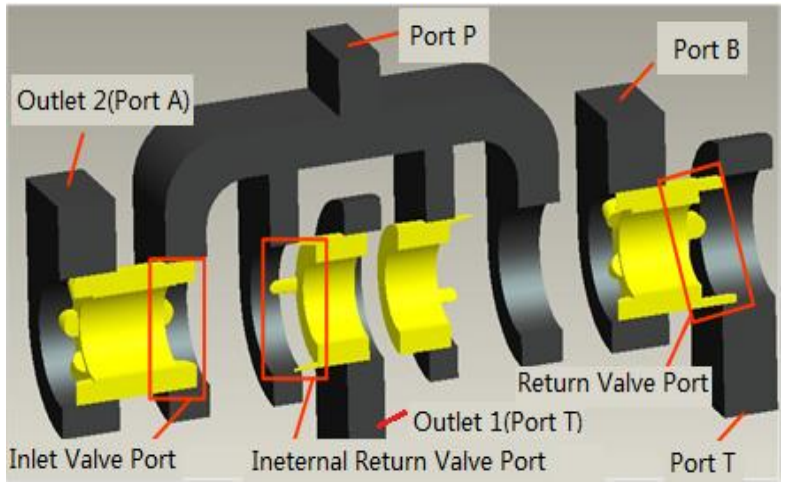

Figure 2. Channel model between the valve cavity and the spool in bucket valve

Because of the manufacture error, the spool cannot strictly locate at the median, and the process or return hole will open sometimes. By considering various non-linear characteristics comprehensive, the orifice flow generalized equation of the inlet $Q_{1 i}$ can be defined as

$$
Q_{1 i}=\left\{\begin{array}{lc}
0 & x \leq d \\
C_{d} w \sqrt{2 / \rho} \cdot \sqrt{P_{p}-P_{1}}(x-d-b) ; & d<x<b+d \\
C_{d} w \sqrt{2 / \rho} \cdot \sqrt{P_{p}-P_{1}} L & b+d \leq x
\end{array}\right\}
$$

Where, $C_{d}$ - coefficient of flow; $w$ - gradient of valve area; $r$-density of flow $\mathrm{kg} / \mathrm{m}^{3} ; x$ - displacement of spool $m, L$ - width of output port $m$.

As well, the orifice flow generalized equation of the return $Q_{1 o}$ can be defined as

$$
Q_{1 o}=\left\{\begin{array}{ll}
0 & x \leq d t \\
C_{d} w \sqrt{2 / \rho} \cdot \sqrt{P_{1}-P_{T}}(x-d t-b) & d t<x<d t+b \\
C_{d} w \sqrt{2 / \rho} \cdot \sqrt{P_{1}-P_{T}} L ; & d t+b \leq x
\end{array}\right\}
$$

Assuming there is no interior leakage and cavittions when the flow passed the orifice, the total flow of output port 1 is as follows: $Q_{1}=Q_{1 P}-Q_{1 T}$

According to formula (1) and (2), the saturation function is introduced, and the saturation function is defined as formula (3).

$$
v(x, \delta, L)=\left\{\begin{array}{ll}
0 & x \leq d \\
(x-d-b) & ;<x<d+b \\
L ; & d+b \leq x
\end{array}\right\}
$$

Using the saturation function, the orifice flow equations (1) and (2) can be simplified as:

$$
Q_{1}=C_{d} w \sqrt{2 / \rho} \cdot\left(\sqrt{P_{p}-P_{1}} \cdot v(x, d 1, L)-\sqrt{P_{1}-P_{T}} \cdot v(x, d t 1, L)\right)
$$

Analogously, the flow equation of port 2 can be obtained(5):

$$
Q_{2}=C_{d} w \sqrt{2 / \rho} \cdot\left(\sqrt{P_{2}-P_{T}} \cdot v(x, d 2, L)-\sqrt{P_{P}-P_{2}} \cdot v(x, d t 2, L)\right)
$$

Based on formula (4) and (5), all the nonlinear characteristics of the proportional valve can be simulated, especially when the positive overlap, negative overlap and asymmetrical valve face exist in the spool. Their relationship is shown in (6). The characteristic of port 1 and port 2 can be changed by changing parameter $d$ and $d t$.

$$
\begin{cases}d=d t>0 ; & \text { symmetric positive overlap stucture } \\ d=d t<0 ; & \text { symmetric negative overlap stucture } \\ d \neq d t ; & \text { asymmetric overlap stucture }\end{cases}
$$

The model power bond graph of the proportional valve is shown in Fig.2, and the $R_{1}, R_{2}, R_{3}, R_{4}$ are the flowing resistance of ports.

Where "SE" is the potential source, the "half-arrow" means the direction of the positive energy flux, " 0 " knot is the flow knot, the algebraic sum of the flow variables around which is zero, and" 1 "knot is the potential knot, the potential variables around which is zero. The bond and graph are shown in Fig.2. which indicates that the outflow of pump can flow to port 1 and port 2, and the flow sum of port 1 and port 2 is equal to the outflow the pump. The flow from port 1 or port 2 to the executive component is equal to the outflow of the pump subtracted by the quantity of flow into the tank.

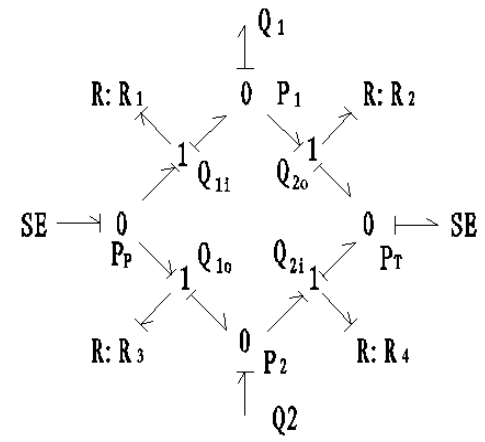

Figure 3. Bond graph of large flow proportional valve

\section{CHARACTERISTIC ANALYSIS OF PROPORTIONAL VALVE}

The uniform flow equations (5) and (6) proposed in section one can be extended to analyze all the hydraulic systems without considering the cover type of spool. And these equations have overcome the problem that the valve 
port flow equation[5] can only be used in the hydraulic system which is strictly centered.

In the hydraulic control system, the ordinary analysis method is to match the precision expensive strictly centered spool with valve body by eliminating the positive and negative overlap. However, the development of nonlinear control theory ${ }^{[6-10]}$ has made the dynamics research about the positive overlap and negative overlap spool become possible, or at least can handle the nonlinear characteristics.

In order to satisfy the application of nonlinear control and obtain the flow characteristics of large flow electrohydraulic proportional valve, some assumptions are defined as:

1) The spool has the same cross area of chamber, that is $A_{1}=A_{2}$;

2) The fluid is uncompressible;

3) The spool structure match with the overlap parameters, that is $d 1=d t 2, d 2=d t 1$;

4) The fluid has no inversion and cavitation, that is $P_{p}>P_{1}>P_{T}>0$;

Based on the above assumptions, especially assumption 1), it is easy to obtain

$Q_{1}=Q_{2}$, then, the flow equations (5) and (6) can be transformed into:

$$
\begin{aligned}
& \left(\sqrt{P_{p}-P_{1}}-\sqrt{P_{1}-P_{T}}\right) \cdot v(x, d 1, L)= \\
& \left(\sqrt{P_{2}-P_{T}}-\sqrt{P_{P}-P_{2}}\right) \cdot v(x, d 2, L)
\end{aligned}
$$

Then

$$
v(x, d 1, L)=\frac{\left(\sqrt{P_{2}-P_{T}}-\sqrt{P_{P}-P_{2}}\right)}{\left(\sqrt{P_{p}-P_{1}}-\sqrt{P_{1}-P_{T}}\right)} ? v(x, d 2, L)
$$

The detailed analysis of two different overlap situations is described as follows:

1) Positive overlap spool $d 1>0, d t 2>0$,so $P_{p}+P_{T}=P_{1}+P_{2}$;

2) Negative overlap and zero overlap spools $d 1 \leq 0, d t 2 \leq 0, \quad-d 2<x<d 1$, and all flow is zero.

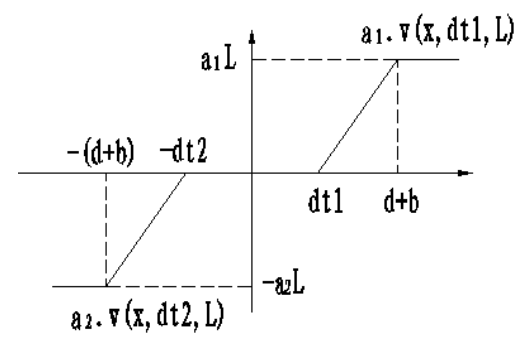

Figure 3. Flow-displacement of positive overlap

By substituting the above pressure relationship into formula (4) and (5), the $Q_{1}$ and $Q_{2}$ can also be written into (9).

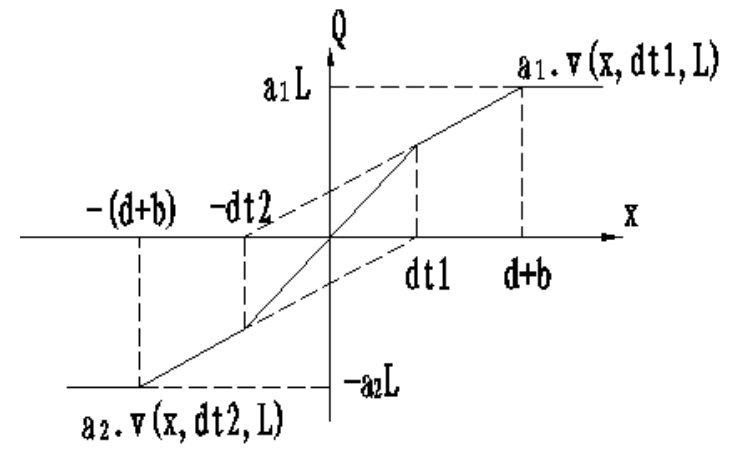

Figure 4. Flow-displacement of negative overlap

$$
\begin{aligned}
& Q_{1}=Q_{1}=C_{d} w \sqrt{2 / \rho} \cdot\left(\sqrt{\frac{P_{p}-P_{1}-\left(P_{1}-P_{2}\right)}{2}}\right. \\
& \left.\cdot v\left(x, \delta_{1}, L\right)-\sqrt{\frac{P_{p}-P_{1}+\left(P_{1}-P_{2}\right)}{2}} \cdot v\left(x, \delta_{2}, L\right)\right)
\end{aligned}
$$

The formula (8) shows the relationship between the flow and spool position when the spool is negative overlap, which can be shown as Fig.3, from which,

$$
\begin{gathered}
\alpha_{2}=C_{d} w \sqrt{2 / \rho} \cdot \sqrt{\frac{P_{p}-P_{1}+\left(P_{1}-P_{2}\right)}{2}} \\
\alpha_{1}=C_{d} w \sqrt{2 / \rho} \cdot \sqrt{\frac{P_{p}-P_{1}-\left(P_{1}-P_{2}\right)}{2}} \text {, it }
\end{gathered}
$$

, it can be seen that the displacement relationship between the flow under the negative overlap of proportional valve and spool displacements can be divided into five different zones.

1) $-d 2<x<d 1$, the flow from the proportional valve to the hydraulic cylinder is zero, and $P_{p}+P_{T} \neq P_{1}+P_{2}$

2) $x \leq-d 2$ or $x \geq d 1$, the flow from the proportional valve to the hydraulic cylinder is proportional to the spool displacement, and the flow quantity can be confirmed by formula (9).

Likewise, the relationship between the flow and spool position when the spool is positive overlap can be obtained (as shown in Fig.4), which can also be divided into five zones.

1) $-d 2<x<d 1$, the flow from the proportional valve to the hydraulic cylinder is not zero, and $v(x, d 1, L)=x+d 1, \quad v(x, d 2, L)=x-d 2$;

2) $x \leq-d 2$ or $x \geq d 1$, the flow from the proportional valve to the hydraulic cylinder is proportional to the spool displacement, which is similar to the situation of negative overlap.

Then, the relationship between the flow and spool displacement when the spool is positive overlap can be written as (10):

$$
\begin{aligned}
& Q_{1}=Q_{1}=C_{d} w \sqrt{2 / \rho} \cdot\left(\sqrt{\frac{P_{p}-P_{1}-\left(P_{1}-P_{2}\right)}{2}}\right. \\
&\left.\cdot(x+d 1)-\sqrt{\frac{P_{p}-P_{1}+\left(P_{1}-P_{2}\right)}{2}} \cdot(x-d 2)\right) \\
&=\left(\alpha_{1}+\alpha_{2}\right) \cdot x+\left(\alpha_{1} \cdot d 1+\alpha_{2} \cdot d 2\right)
\end{aligned}
$$

Where, $\left(a_{1}+a_{2}\right) ? x$ is defined as the flow gain of the proportional valve which is named as $K_{q}$, which is an 
important parameter for the positive overlap zone in the design of hydraulic control system. In the work zone, the change of flow gain will induce the hydraulic control system to be unstable, and it also will determine the gain of open loop hydraulic system. According to formula (9), even if the spool is located at the centre, i.e., $x=0$, there still exists flow output and hydro-cylinder movement. This situation is consistent with the actual positive overlap proportional valve.

The flow gain can be defined as (11):

$$
\begin{aligned}
& K_{q}=\frac{\partial Q}{\partial x}=\left(\alpha_{1}+\alpha_{2}\right)=C_{d} w \sqrt{2 / \rho} / \sqrt{2} \\
& \cdot\left(\sqrt{P_{p}-P_{1}-\left(P_{1}-P_{2}\right)}-\sqrt{P_{p}-P_{1}+\left(P_{1}-P_{2}\right)}\right)
\end{aligned}
$$

Because $\left(P_{1}-P_{2}\right) \leq P_{p}-P_{1}$, the variation range of flow gain $K_{q}$ can be obtained.

\section{EXPERIMENT INVESTIGATION OF LARGE FLOW ELECTRO-HYDRAULIC PROPORTIONAL VALVE}

\section{A. Experimental scheme}

In section two, the flow characteristics in different spool overlap are investigated, and the uniform equation of positive, negative and zero overlap is obtained in theory. In this section, the experiments are employed to validate and amend the flow-displacement characteristic of electrohydraulic proportional valve.

All parameters used in experiment are the same as those used in the simulation.

The experimental system is shown in Fig .6 The control voltage signal is generated by the signal generator, and loaded at the magnet coil of electro-hydraulic valve to make the spool produce displacement after processing by the amplifier. Because of the opening between the spool and valve body, the output control flow can be obtained, and return to the oil tank through the flow meter. By changing the value and direction of the input signal voltage, the flow value and direction can be changed. In order to ensure the accurate position of the spool, the position sensor is employed to detect the position error. Data acquisition experimental field is shown in Fig.7.

The experimental system consists of two one-way flow meters, two relief valves and two pressure gages. When the signal generator makes the proportional valve be located at the left side, the oil provide by the pump will flow from port $\mathrm{P}$ to port $\mathrm{A}$ after passing through the proportional valve. The return pressure can be adjusted by relief valve 2 to change the pressure difference between the front and rear of the valve. When the signal generator makes the valve be located at the right side, the flow will be detected by another oil circuit before returning to the oil tank.
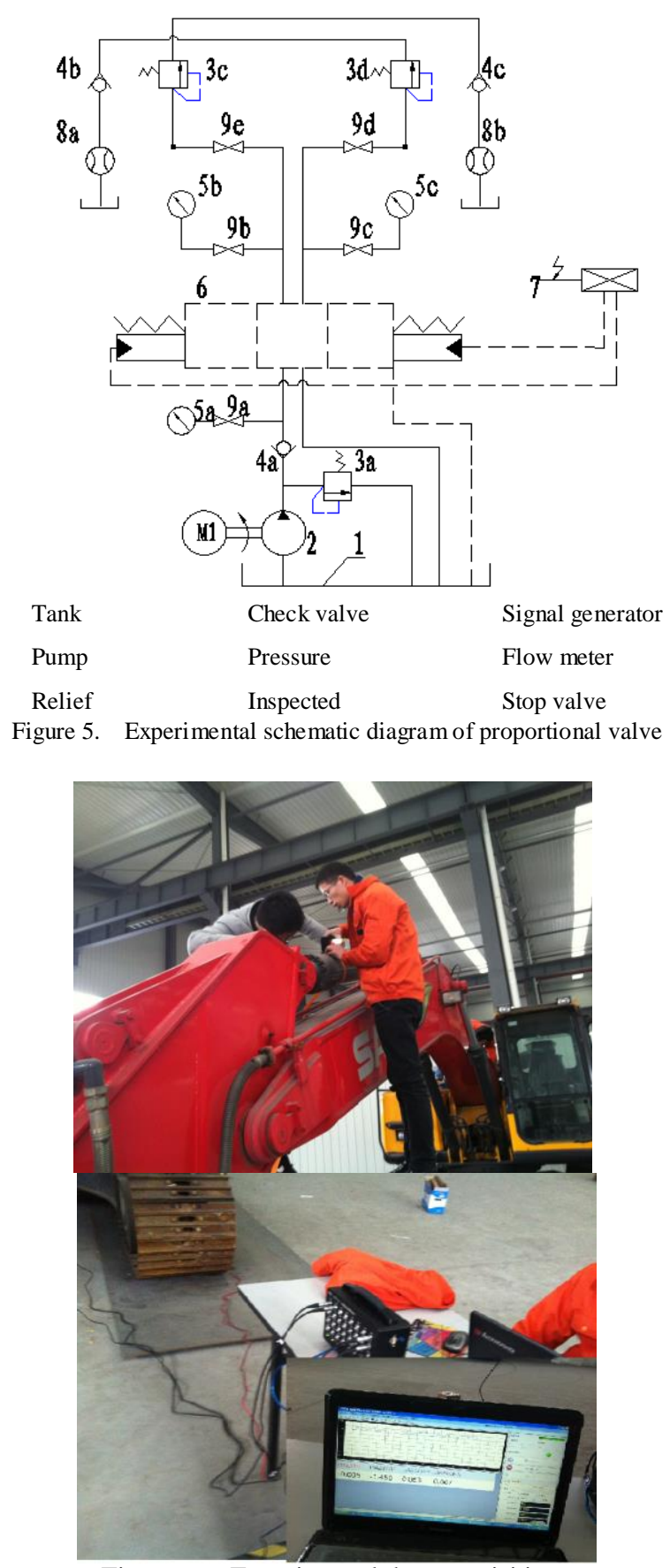

Figure 6. Experimental data acquisition

\section{B. Experimental method and results}

In the measurement, the pressure of return circuit is adjusted by the relief valve. When the pressure difference of the proportional valve is $2.7 \mathrm{Mpa}$, and the viscosity is 30 , the flow values during the process that the input signal increases from zero to the maximum and decreases from the maximum to zero are recorded by the flow meter. The signal generated by the generator is the square wave whose magnitude and frequency are $5 \mathrm{~V}$ and $20 \mathrm{~Hz}$, respectively. The voltage signal fed back from the spool displacement sensor is tested. The feedback signal of the displacement sensor and input signal of the signal generator are read through the oscilloscope.

The flow-displacement relationship of positive overlap and negative overlap is shown in Fig.11 and Fig.12, from 
which, it can be seen that the experiment results are consistent with the theory analysis in the section two.

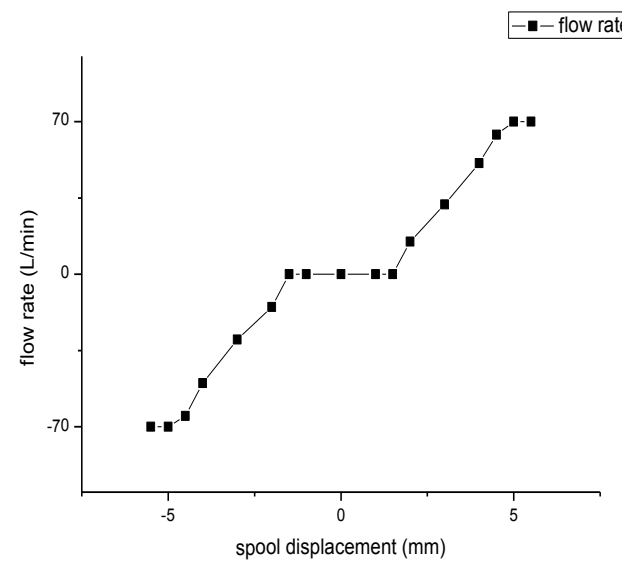

Figure 7 . Flow-displacement of positive overlap

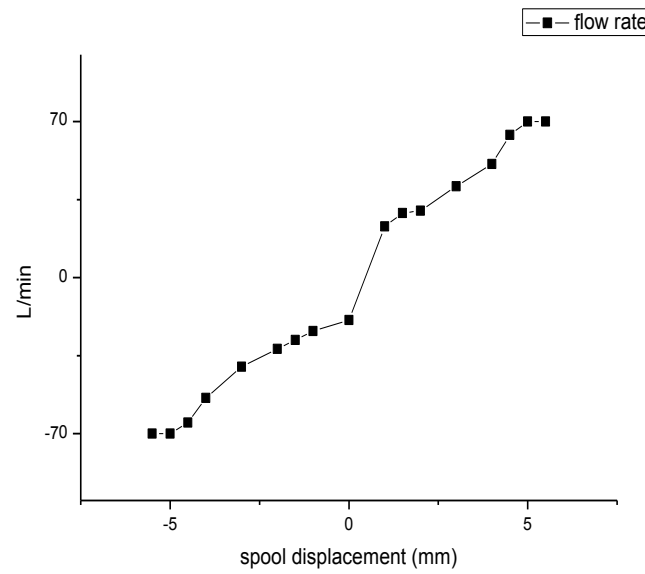

Figue 8 . Flow-displacement of negative overlap

\section{CONCLUSIONS}

According to the orifice flow equation, the uniform model for the large flow proportional valve is established, and the flow characteristics of the proportional valve with different spool overlaps are analyzed. The mathematical equations of the flow which continuously and nonlinearly passes through the port of proportional valve with different spool overlaps are constructed.

From the viewpoint of the experimental scheme, method and results, the flow characteristics of the proportional valve are investigated. The results show that, for positive overlap, the flow input into the hydraulic tank is proportional to the displacement of the spool in the dead zone. For negative overlap, even though the spool is located at the median, the flow output also exists, which then induces the piston movement of hydraulic tank. This situation is consistent with the non-matching overlap response of actual negative proportional valve.

\section{ACKNOWLEDGMENT}

This work is supported in part by The National Natural Science Fund, Jiangsu Natural Science Foundation, University of Jiangsu Natural Science Foundation, Nanjing Institute of Technology and SANY Co., Ltd in Jiangsu. Lecturer, Support Fund No. 11302097, BK20130741, 13KJB460009, QKJA201201.

\section{REFERENCES}

[1] Leandro dos Santos Coelho, Mauro André Bar-bosa Cunha. Adaptive cascade control of a hydraulic actuator with an adaptive dead-zone compensation and optimization based on evolutionary algorithms[J],Expert Systems with Applications, 2011,38(10):12262-12269.

[2] K. Dasgupta, J. Watton. Dynamic analysis of proportional solenoid controlled piloted relief valve by bondgraph Original Research Article[J], Simulation Modelling Practice and Theory, 2005,13(1):21-38

[3] Arkan Kayihan, Francis J. DoyleIII. Friction compensation for a process control valve Original Research Article[J], Friction compensation for a process control valve,2000,8(7): 799-812.

[4] [4]Bora Eryilmaz, Bruce H. Wilson. Unified model-ing and analysis of a proportional valve Original Research Article[J] Journal of the Franklin Institute, 2006,343(1):48-68.

[5] Chasik Park, Honghyun Cho, Yongtaek Lee, Yongchan Kim. Mass flow characteristics and em-pirical modeling of R22 and R410A flowing through electronic expansion valves Original Research Article[J], 2007,30(8):1401-1407.

[6] B. Scholz-Reiter, M. Freitag, A. Schmieder. Modelling and Control of Production Systems based on Nonlinear Dynamics Theory Original Research Article[J],CIRP Annals - Manufacturing Technology, 2002,51(1):375-378.

[7] Ahmad M. Harb, Ashraf A. Zaher. Nonlinear control of permanent magnet stepper motors Origi-nal Research Article[J],Communications in Nonlinear Science and Numerical Simulation, 2004,9(4):443-458.

[8] S. Effati, A.R. Nazemi.A new method for solving a system of the nonlinear equations Original Research Article[J],Applied Mathematics and Computation,2005,168(2):877-894.

[9] Henry C L, Craig V S J. Measurement of no-slip and slip boundary conditions in confined Newtonian fluids using atomic force microscopy[J]. Physical Chemistry Chemical Physics, 2009, 11: 9514-9521.

[10] Neto C, Evans D R, Bonaccurso E, Butt H J, Craig V S J Boundary slip in Newtonian liquids: a review of experimental studies[J]. Reports on Progress in Physics, 2005, 68: 2859-2897. 